\title{
1 The influence of non-neural factors on BOLD signal magnitude
}

2 Jan W. Kurzawski ${ }^{1}$, Omer Faruk Gulban ${ }^{2,3}$, Keith Jamison ${ }^{4}$, Jonathan Winawer ${ }^{1^{*}}$, Kendrick $3 \mathrm{Kay}^{5^{*}}$

4

5

6

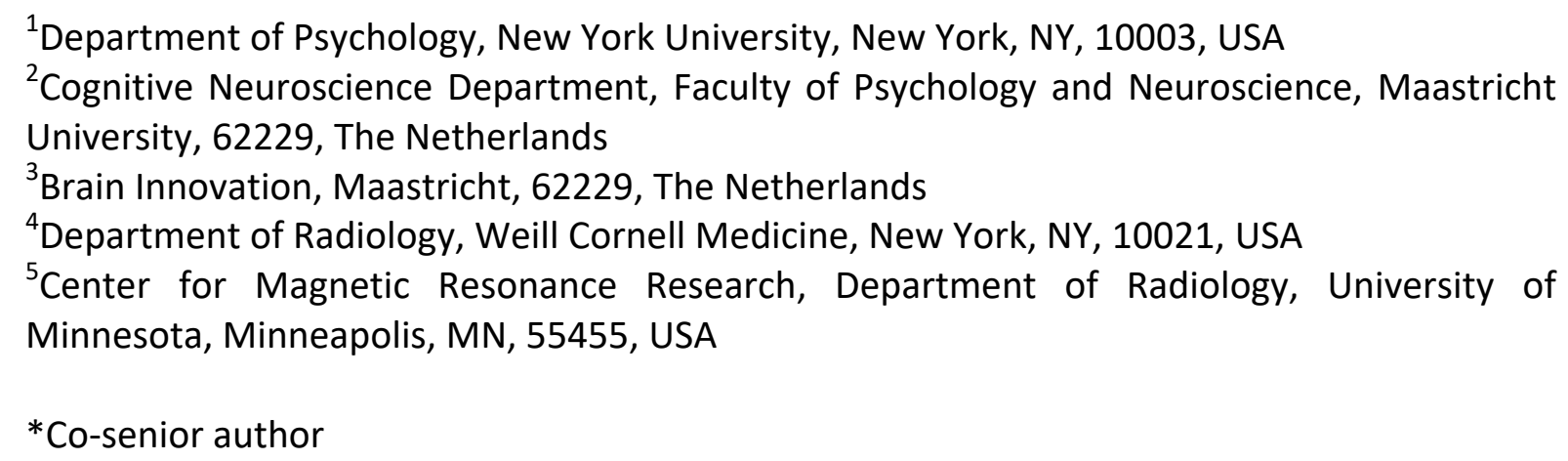

Corresponding Author: Jan W. Kurzawski, Department of Psychology, 6 Washington Place, New York University, NY, 10003 (jan.kurzawski@nyu.edu)

Keywords: BOLD signal, fMRI, vasculature, horizontal meridian, vertical meridian, cerebral sinus.

\section{Number of pages: 25}

Number of figures: 10

Number of words for: abstract | introduction | discussion: 238/250 | 648/650 | 1427/1500

Conflict of interest: The authors declare no competing financial interests.

Acknowledgments: This research was funded by the US National Eye Institute R01EY027964, R01-MH111417 and R01-EY027401 to JW. Collection of the NSD dataset was supported by NSF CRCNS grants IIS-1822683 (to KK) and NSF IIS-1822929. 
43

44

45

46

47

48

49

50

51

52

53

54

55

56

57

58

59

60

61

62

63

64

65

66

67

68

69

70

71

72

73

\section{Introduction}

\section{Abstract}

To what extent is the size of the blood-oxygen-level-dependent (BOLD) response influenced by factors other than neural activity? In a re-analysis of three neuroimaging datasets, we find large systematic inhomogeneities in the BOLD response magnitude in primary visual cortex (V1): stimulus-evoked BOLD responses, expressed in units of percent signal change, are up to $50 \%$ larger along the representation of the horizontal meridian than the vertical meridian. To assess whether this surprising effect can be interpreted as differences in local neural activity, we quantified several factors that potentially contribute to the size of the BOLD response. We find strong relationships between BOLD response magnitude and cortical thickness, cortical curvature, and the presence of large veins. These relationships are consistently found across subjects and suggest that variation in BOLD response magnitudes across cortical locations reflects, in part, differences in anatomy and vascularization. To compensate for these factors, we implement a regression-based correction method and show that after correction, BOLD responses become more homogeneous across V1. The correction reduces the horizontal/vertical difference by about half, indicating that some of the difference is likely not due to neural activity differences. Additionally, we find that while the cerebral sinuses overlap with the vertical meridian representation in $\mathrm{V} 1$, they do not explain the observed horizontal/vertical difference. We conclude that interpretation of variation in BOLD response magnitude across cortical locations should consider the influence of the potential confounding factors of cortical thickness, curvature, and vascularization.

\section{Significance statement}

The magnitude of the BOLD signal is often used as a surrogate of neural activity, but the exact factors that contribute to its strength have not been studied on a voxel-wise level. Here, we examined several anatomical and measurement-related factors to assess their relationship with BOLD magnitude. We find that BOLD magnitude correlates with cortical anatomy and macrovasculature. To remove the contribution of these factors, we propose a simple, data-driven model that can be used in any functional magnetic resonance imaging (fMRI) experiment. After accounting for the confounding factors, BOLD magnitude becomes more spatially homogenous. Our correction method improves the ability to make more accurate inferences about local neural activity from fMRI data.

The blood-oxygen-level-dependent (BOLD) signal measured by fMRI is an important tool for non-invasive study of the human nervous system. However, the neural mechanisms underlying BOLD remain an active area of investigation (Herman et al., 2017). One clear conclusion is that the BOLD signal is strongly influenced by neural activity (Arthurs et al., 2000; Heeger et al., 2000; Attwell and ladecola, 2002; Heeger and Ress, 2002; Logothetis, 2002; Lee et al., 2010; Siero et al., 2014). For a given location in the brain, and within a constrained paradigm (e.g., viewing different images and measuring the response that they elicit in visual cortex), the BOLD signal magnitude appears to be lawfully related to basic 
measures of neural activity. For example, as stimulus contrast increases, neural firing rates and BOLD magnitude increase in proportion (Heeger et al., 2000). Similarly, increase in coherence of stimulus motion boosts BOLD magnitude and firing rates in V5/MT (Britten et al., 1993; Rees et al., 2000). When comparing different experimental paradigms or different brain locations, however, it is less clear how to interpret differences in the magnitude of the BOLD signal. For example, seeing a stimulus and expecting a stimulus can both elicit robust BOLD signals in V1, but the underlying neural activity is very different in the two paradigms (Sirotin and Das, 2009; Herman et al., 2017). It is also the case that similar BOLD signal magnitudes in two locations may be linked to very different underlying neural activity. These two limitations are reviewed by (Logothetis, 2008).

There are several reasons to believe that BOLD signal magnitudes, even within a fixed experimental paradigm, are influenced by factors that are not directly related to neural activity. The BOLD response, quantified as percent signal change above baseline, can be especially high in voxels containing large veins (Menon et al., 1993; Kim et al., 1994; Hoogenraad et al., 1999; Kay et al., 2019) or unusually low, delayed, and/or displaced in voxels near cerebral sinuses (Winawer et al., 2010; Jamison et al., 2017). The choice of MRI sequence, field strength (van der Zwaag et al., 2009), and sequence parameters like echo time (Gorno-Tempini et al., 2002) can also affect BOLD signal magnitude, and these effects may vary across the brain (Herman et al., 2017). Indeed, it has been reported that BOLD may vary across the cortex up to $40 \%$ simply due to different orientation of vasculature relative to the direction of the static magnetic field (Gagnon et al., 2015; Gagnon et al., 2016). Furthermore, recent high-resolution fMRI studies have shown that BOLD signal magnitude clearly depends on cortical depth. It is highest in the superficial layers which are positioned near large pial veins and decreases with cortical depth (Polimeni et al., 2010; Koopmans et al., 2011; Zimmermann et al., 2011; Yu et al., 2014; Fracasso et al., 2016a; Fracasso et al., 2016b; Dumoulin, 2017; Dumoulin et al., 2018; Kay et al., 2019; Self et al., 2019; van Dijk et al., 2020).

In this paper, we study variations in BOLD signal magnitude within a fixed paradigm, focusing our efforts on primary visual cortex (V1). We believe that by focusing on a single brain region in well-controlled visual paradigms, we are in the best position to derive sound interpretations of differences in BOLD signal magnitudes across the cortex. In three distinct datasets, we demonstrate large differences between the meridional locations: the BOLD magnitude in V1 is up to $50 \%$ higher along the representation of the horizontal meridian than along the representation of the vertical meridian. We then investigate the potential basis of these inhomogeneities by analyzing factors that are in principle distinct from neural activity. We find that several of these factors are systematically related to observed variation in BOLD magnitudes across V1. To remove their influence, we propose a simple correction method and show that the correction increases BOLD signal homogeneity across $\mathrm{V} 1$, reducing the horizontal-vertical difference by about half. 


\section{Methods}

\section{Datasets}

We used three publicly available visual fMRI datasets: the Human Connectome Project (HCP) 7T Retinotopy Dataset (Benson et al., 2018), the Natural Scenes Dataset (NSD) (Allen et al., 2021), and the Temporal Decomposition Method (TDM) Dataset (Kay et al., 2020). All data were acquired on 7T MR scanners using gradient-echo pulse sequences (technical details provided in Supplementary Table 1). The datasets varied in stimulus properties and experimental design. HCP stimuli consisted of rings, wedges, and bars in a retinotopic mapping experiment; NSD stimuli consisted of natural scene images; and TDM stimuli consisted of high-contrast rings presented at different eccentricities. Experimental details are shown in Figure 1. The analyses performed in this paper start with pre-processed data from each dataset (details below).
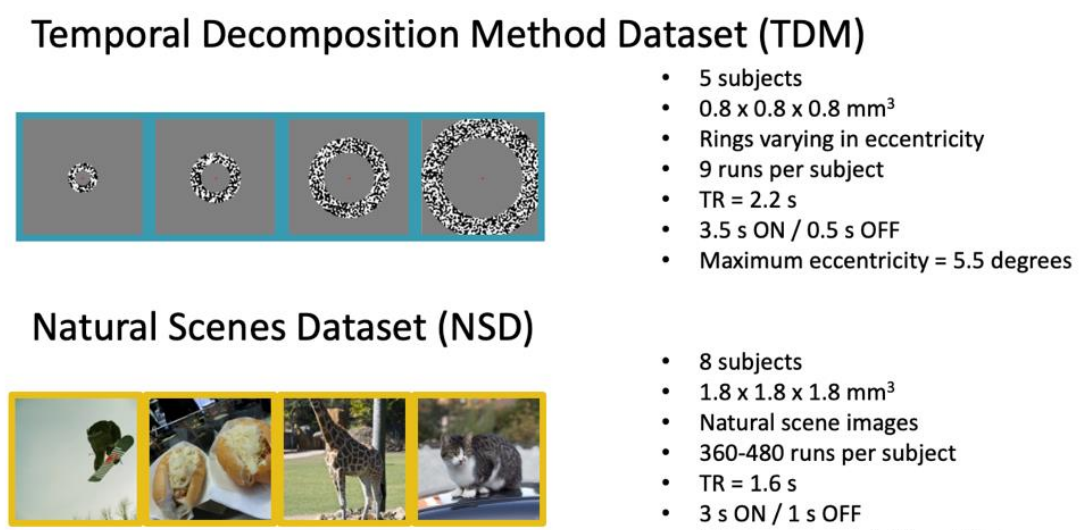

- 8 subjects

- $1.8 \times 1.8 \times 1.8 \mathrm{~mm}^{3}$

- Natural scene images

- 360-480 runs per subject

- $\mathrm{TR}=1.6 \mathrm{~s}$

- 3 s ON / 1 s OFF

- Maximum eccentricity $=4.2$ degrees

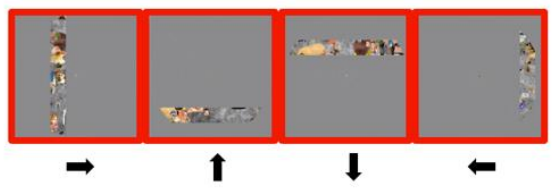

- 181 subjects

- $1.6 \times 1.6 \times 1.6 \mathrm{~mm}^{3}$

- Textures viewed through spatial apertures

- 6 runs per subject

- $T R=1 \mathrm{~s}$

- Continuous design

- Maximum eccentricity $=8$ degrees

Figure 1 - Datasets used in this study. Stimulus images for each of the datasets are shown. For TDM, stimuli consisted of 6 rings varying in eccentricity. For NSD, stimuli consisted of natural scene images. For HCP, the experiment consisted of several retinotopic mapping runs that included expanding and contracting rings, rotating wedges, and moving bars filled with a colorful object-based texture. Additional acquisition details are provided in Supplementary Table 1.

\section{Extracting BOLD magnitude}

From each dataset, we extracted a measure of BOLD signal magnitude at each cortical surface vertex. For TDM, we started with the pre-processed $\mathrm{FMRI}$ time-series data provided 
used these regressors with GLMdenoise (Kay et al., 2013b) to estimate a beta weight for each condition. We computed the maximum beta weight across all conditions for each voxel as the measure of BOLD signal magnitude. These results are defined at six different cortical depths (equidistant from $10 \%$ to $90 \%$ of the cortical depth) in each subject's native surface space. (Depth assignment was achieved by a spatial interpolation of each fMRI volume at the locations of the six depth-dependent cortical surfaces; see Kay et al. (2020) for details.) For NSD, we took the 'meanbeta' values ( $1 \mathrm{~mm}$ data preparation, beta version 2 ) provided with the dataset; these values indicate the average BOLD percent signal change observed across all stimulus trials and all scan sessions. We then mapped these values to the 3 cortical depth surfaces provided in NSD (positioned at $25 \%, 50 \%$, and $75 \%$ of the cortical depth), and then averaged across depth to produce one value per surface vertex in the subjects' native surface space. The HCP dataset was previously analyzed (Benson et al., 2018) with a population receptive field (pRF) model (Dumoulin and Wandell, 2008) implemented in analyzePRF (Kay et al., 2013a). The model includes a gain parameter that describes the amplitude of the BOLD response of a given voxel (or vertex) to the object-based texture (covering the entire $\mathrm{pRF}$ ) for a single repetition time (TR=1s). We quantified BOLD in terms of percent signal change (\%BOLD) by dividing the gain parameter by mean signal intensity and multiplying by 100 . The results are prepared in FreeSurfer's fsaverage space.

\section{Visual field mapping}

We used retinotopic mapping to divide the primary visual cortex into a set of regions. For $\mathrm{HCP}$, we used polar angle and eccentricity estimates available from the data release. For the TDM and NSD datasets, we mapped Benson's polar angle and eccentricity atlas using neuropythy software (Benson and Winawer, 2018). We use the following convention for all 3 datasets: the upper vertical meridian corresponds to $0 \mathrm{deg}$, the horizontal meridian corresponds to $90 \mathrm{deg}$, and the lower vertical meridian corresponds to $180 \mathrm{deg}$. Note that the polar angle estimates are rescaled for the correlation and linear regression analysis (see next section). We used Benson's definition of the extent of visual areas V1, V2, and V3 for all 3 datasets (Benson et al., 2014).

\section{Quantification of non-neural factors}

For each dataset, we quantified a number of factors that might be related to variation in the magnitude of the BOLD signal across cortical locations. These factors include the following:

1. Cortical curvature was obtained from FreeSurfer outputs (Dale et al., 1999; Fischl and Dale, 2000), and refers to the geometry of the folding pattern of the cortical surface. Negative values correspond to gyri while positive values correspond to sulci. Curvature is quantified as $1 / r$, where $r$ is the radius of an inscribed circle measured in $\mathrm{mm}$.

2. Cortical thickness was also obtained from FreeSurfer outputs. It is measured in $\mathrm{mm}$ and corresponds to the distance between the outermost (close to cerebrospinal fluid) and innermost (close to white matter) boundaries of gray matter.

3. Angle with respect to $B O$ was calculated by considering the angle (theta) between the pial surface normal and the direction of the $B_{0}$ static magnetic field as estimated 
from NIFTI header information. Angle was quantified in degrees and was normalized as abs(theta-90) such that a final value of 0 deg indicates that the cortical surface is parallel to the magnetic field and a final value of 90 deg indicates that the cortical surface is perpendicular to the magnetic field.

4. RF coil bias was taken to be the result of fitting a 3D polynomial to the mean signal intensity in the fMRI data. The values are in raw scanner units. This method has been previously used (Kay et al., 2019).

5. Mean bias-corrected EPI was calculated as the mean signal intensity in the fMRI data divided by the RF coil bias (Kay et al., 2019). The units range from approximately 0 to 2 , and indicate percentages (e.g., 0.5 means $50 \%$ of the strength of typical signal intensities).

6. Polar angle (theta) was obtained from Benson's atlas (Benson et al., 2014) and normalized as abs(theta-90). After this normalization, 0 deg corresponds to the horizontal meridian, while 90 deg corresponds to the upper and lower vertical meridians.

7. Depth was estimated (in the TDM dataset) by generating 6 cortical surfaces equally spaced between $10 \%$ and $90 \%$ of the distance from the pial surface to the boundary between gray and white matter. These surfaces are numbered from 1-6, where 1 is outermost and 6 is innermost.

\section{Definition of regions of interest}

Using the visual field mapping results, we defined regions of interest (ROIs) corresponding to the representation of the horizontal and vertical meridians within V1. The ROIs were defined by limiting the eccentricity to the maximum stimulus eccentricity used in each dataset and limiting the angle to a specific range (e.g., to create a V1 ROI for the upper vertical meridian with a width of $20 \mathrm{deg}$, we created a mask where polar angle estimates were higher than 0 and lower than 20).

\section{Modelling variation in BOLD signal magnitude}

To account for non-neural contribution to \%BOLD, we used a multiple regression model. The modeled data $(Y)$ consisted of the \%BOLD value observed at each surface vertex in visual areas V1-V3. Although this study focuses on BOLD homogeneity in V1, we include \%BOLD in $\mathrm{V} 1-\mathrm{V} 3$. This is because we are attempting to establish relationships that might generalize across different cortical regions. Furthermore, if we were to include only vertices in V1, we would be at high risk of removing genuine neural activity differences (e.g. those that may exist between the horizontal and vertical meridians) that correlate with the non-neural factors.

The variables used to model the data included cortical thickness, cortical curvature, and mean bias-corrected EPI intensity. (Only these three factors showed evidence of being substantially related to BOLD magnitude; see Results.) The variables were standardized (zscored) and, together with a constant term, were included as predictors in the design matrix $(X)$. Ordinary least-squares estimates for beta weights were obtained in the following linear model: 


$$
Y=X \beta+n
$$

where $Y$ is the \%BOLD magnitude at each vertex, $X$ is the 4-column design matrix, $\beta$ is a set of beta weights (4 per vertex), and $n$ is a set of residuals.

\section{Major cortical sulci}

In several figures we show outlines of major cortical sulci. These include the calcarine sulcus (CALC), parieto-occipital sulcus (POS), intraparietal sulcus (IPS), occipitotemporal sulcus (OTS), and superior temporal sulcus (STS). These sulci were manually labelled on the fsaverage surface and then mapped to each individual's native surface.

\section{Atlas of cerebral sinuses}

The cerebral sinuses are the largest draining veins in the brain and are known to cause artifacts in fMRI data (Winawer et al., 2010). To aid in identifying the location of the sinuses, we first created an atlas of the sinuses using the T1- and T2-weighted volumes from the HCP1200 dataset (Van Essen et al., 2012). Specifically, we performed the following steps:

1) We obtained T1-weighted and T2-weighted volumes from the 494 subjects from the HCP1200 dataset for which twin pairings are available. We used the versions of these volumes that are already nonlinearly transformed to MNI space.

2) Because the sinuses have high intensity (bright) in T1-weighted volumes and low intensity (dark) in T2-weighted volumes, we divided T1 intensity values by T2 intensity values to accentuate the locations of the sinuses. We refer to the resulting ratios as the sinus map.

3) The sinus map from each subject was intensity normalized, trimmed for outliers, and averaged across subjects to produce a group-average sinus map (Figure $\mathbf{2 A}$ ).

4) We manually defined four control points on the group-average sinus map that mark the geometrical termination of the four sinus segments, namely, the superior sagittal sinus, the straight sinus, and the left and right transverse sinus. Using the fastmarching method (Sethian, 1996), we traced paths that follow the sinus between the control points and used the branching of these paths to define the sinus segments (Figure 2B).

The primary outputs of this atlas creation procedure are 1-mm volumes in MNI space that represent likely locations of sinus voxels as well as their segment labels (Figure 2C). These resources may be useful for other studies and are freely available at https://osf.io/2nc4x/. In the current study, we used the sinus atlas to identify cortical locations that may be affected by sinus artifacts. Specifically, we prepared a binary version of the sinus atlas in the native volumetric space of a given subject and then blurred this binary volume with a Gaussian kernel with full-width-half-maximum of $5 \mathrm{~mm}$. This enables identification of cortical gray matter positioned at varying distances from the sinuses. 
A

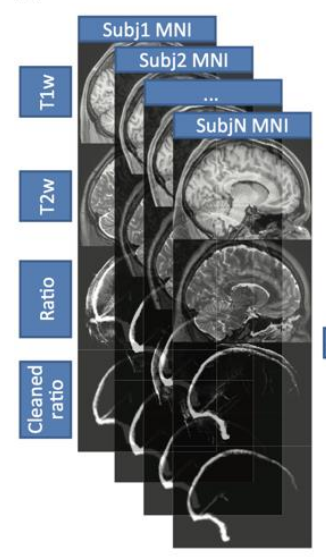

B

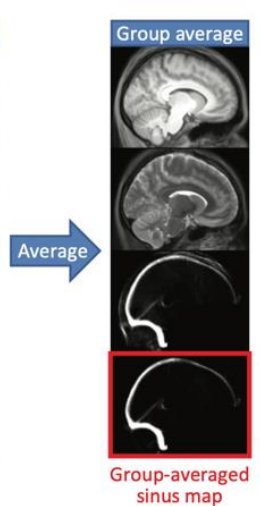

\section{C}

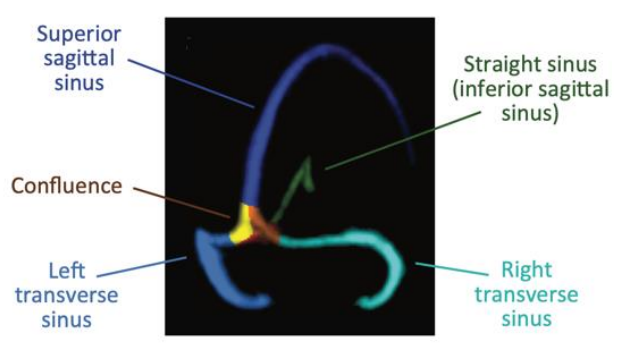

Figure 2 - Constructing an atlas of the cerebral sinuses. This figure illustrates our procedure for creating a sinus atlas using T1- and T2-weighted volumes prepared in MNI space. A) Procedure for creating a groupaveraged sinus map. B) Procedure for finding the branches of the sinuses. C) The final representation of the sinus atlas. Different colors show different branches of the sinuses. The atlas is freely available at https://osf.io/2nc4x/.

\section{Data and code availability}

The datasets that are used in this paper are freely available online: NSD (http://naturalscenesdataset.org), HCP (https://osf.io/bw9ec/), and TDM (https://osf.io/j2wsc/). Code that reproduces the main figures in this paper is available at https://github.com/jk619/meridianbias/. Associated data files as well as the sinus atlas are available at https://osf.io/2nc4x/.

\section{Results}

\section{Stronger BOLD responses along the V1 horizontal meridian}

We examined BOLD response magnitudes in three freely available datasets: the Natural Scenes Dataset (NSD; Allen et al., 2021), the data used for the Temporal Decomposition Method (TDM; Kay et al., 2020), and the Human Connectome Project 7T Retinotopy Dataset (HCP; Benson et al., 2018). Each dataset contains BOLD responses to different types of visual stimulation (see Methods). We defined one region of interest (ROI) for the horizontal meridian (HM) and one for the vertical meridian (VM) (Figure 3A-B). These ROls represent a wedge-shaped region in the visual field centered at the horizontal meridian with a width of 40 deg (horizontal) and two wedges abutting the vertical meridian each with a width of 20 deg (vertical).

In each of the three datasets, we compared BOLD magnitudes expressed in percent signal change (\%BOLD) observed for the VM with BOLD magnitudes for the HM (Figure 3C-E). In each dataset, we find higher \%BOLD in the HM ROls compared to the VM ROIs. We summarize this difference with an asymmetry ratio: (HM-VM)/mean(HM,VM). All datasets 
show strong asymmetry, with an asymmetry ratio of $\sim 30 \%$. Positive values for the asymmetry ratio indicate greater response for the horizontal meridian.
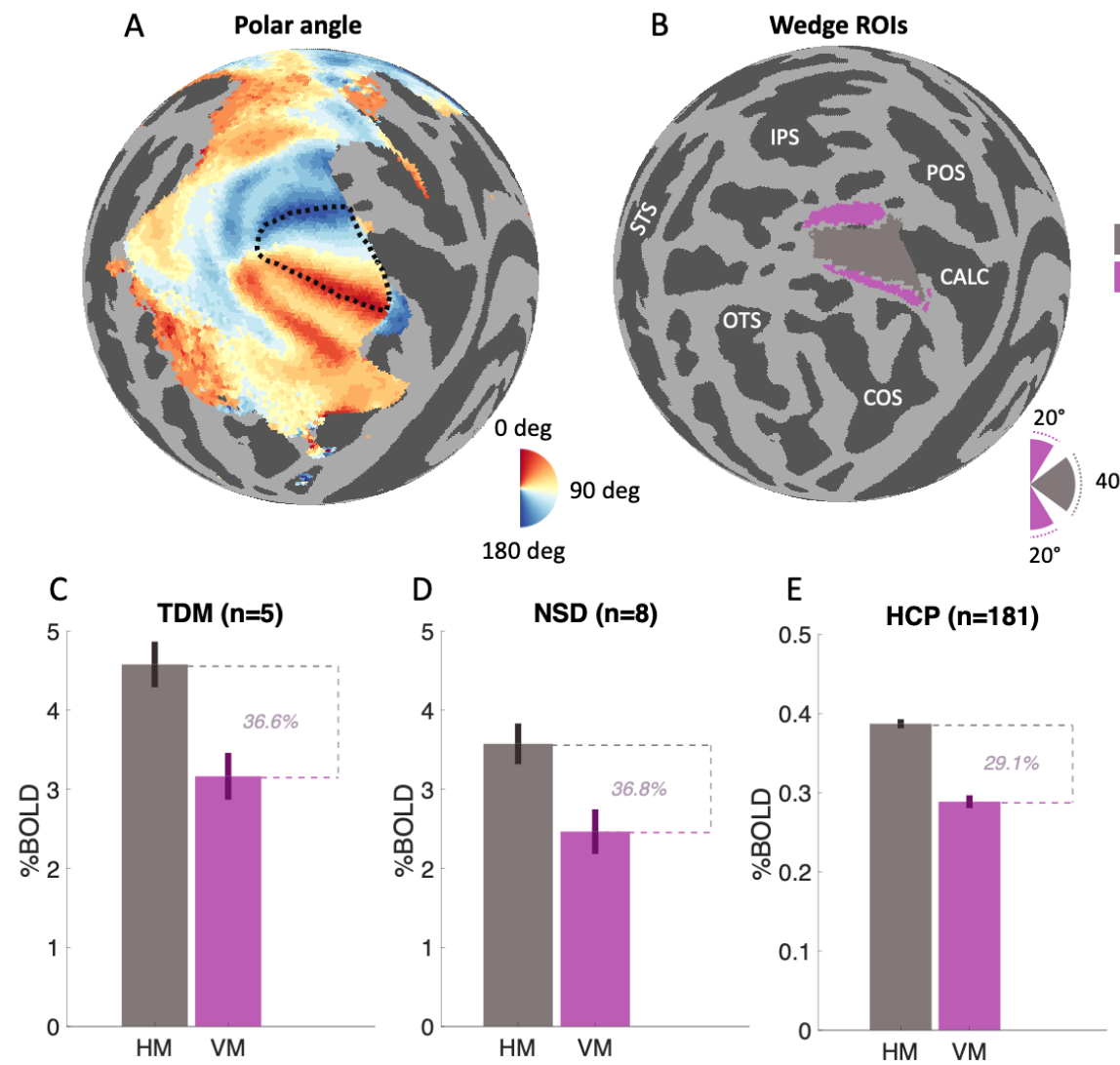

Horizontal Meridian (HM)

Vertical Meridian (VM)

Figure 3 - BOLD magnitude is higher at the horizontal meridian in V1. A) Polar angle map of group-average HCP subject (999999) with V1 boundary outlined in dotted black lines. B) Horizontal and vertical regions of interest (ROIs) are indicated in gray and magenta, respectively. White text indicates major brain sulci (see Methods). C-E) Mean BOLD magnitude for horizontal and vertical ROIs in the three datasets. Error bars indicate standard error across subjects.

One possibility is that the horizontal and vertical V1 BOLD responses are in fact similar, but the vertical ROIs appear to have lower signal due to mixing with signal from V2. V2 and V1 border along the vertical meridian representation, and blurring might occur either in acquisition or in pre-processing and analysis. To further our understanding of the V1 response asymmetries, we re-computed asymmetry ratios using smaller wedges at many locations (Figure 4A). While the asymmetry is strongest at the cardinal meridians, some horizontal/vertical asymmetry is found at least 30 deg away from the meridians in all three datasets (Figure 4B). This argues against the explanation that the asymmetry is caused by spillover from V2. 
A

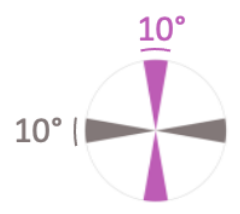

$0^{\circ}$

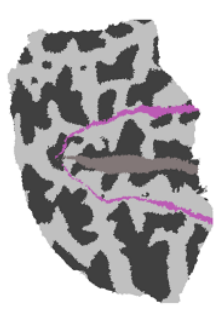

B
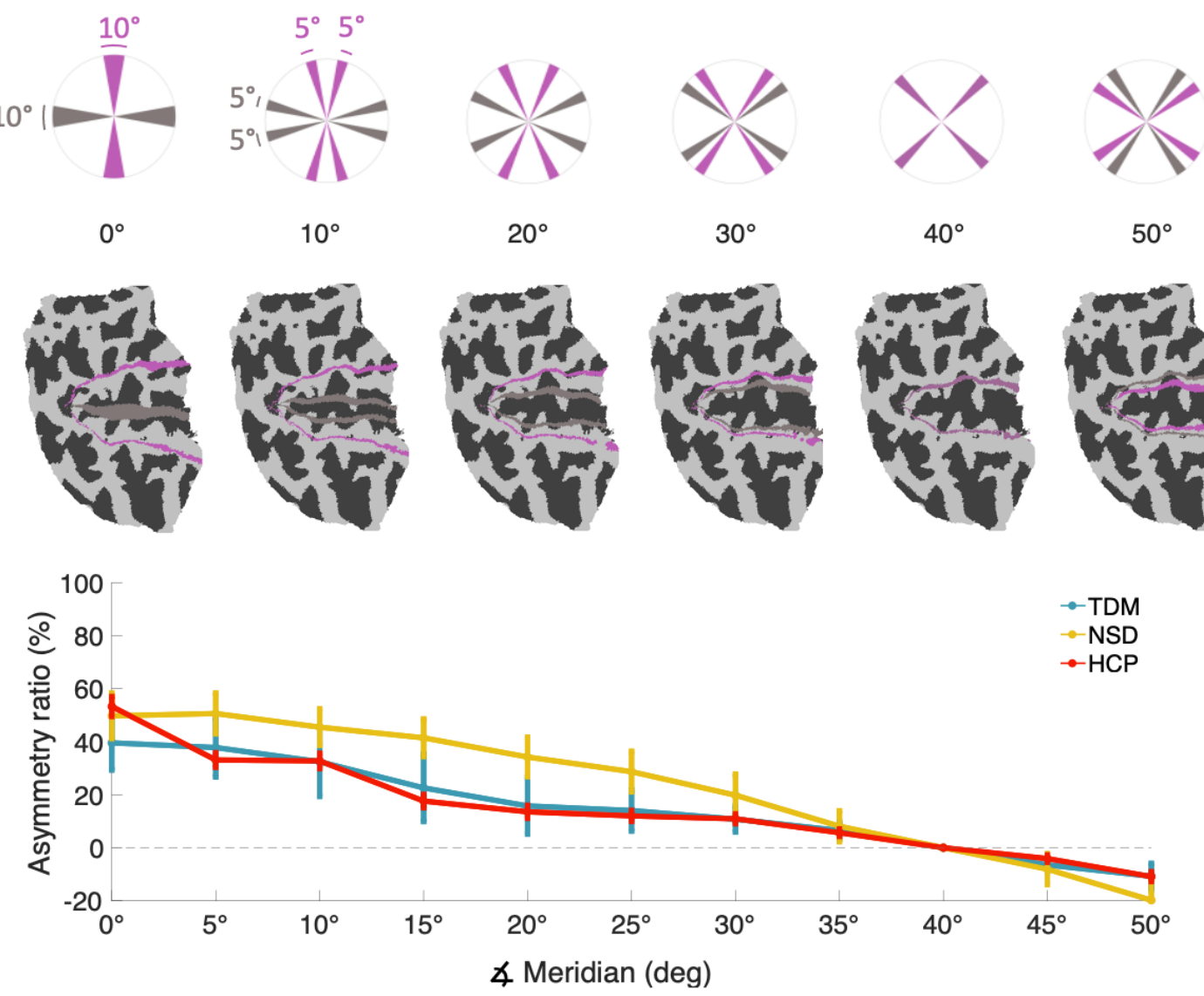

$20^{\circ}$
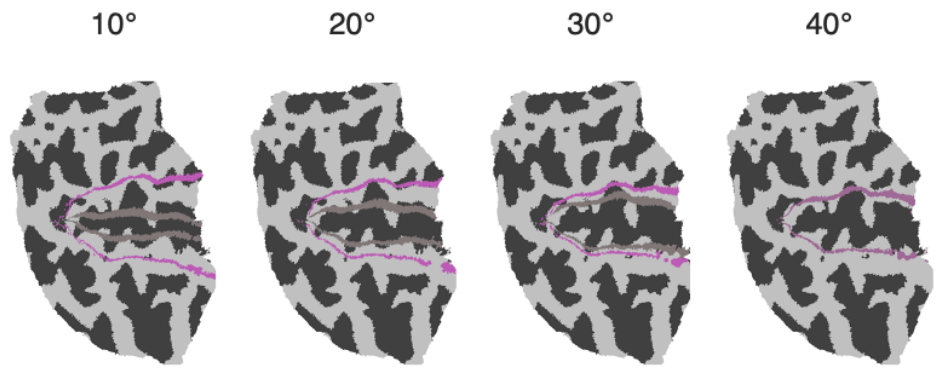

341

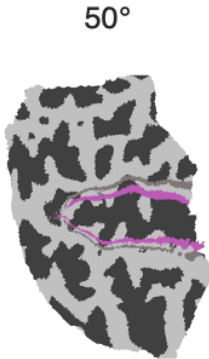

\section{B}

Figure 4 - BOLD asymmetries generalize to off-cardinal locations. To further understand V1 BOLD asymmetry, we manipulated the location of the wedge ROIs in 5-deg increments. A) The upper row shows the visual field location of wedge ROIs and the lower row shows the corresponding cortical locations (flattened left hemisphere). For clarity, we show only every other set of ROIs. B) Asymmetry ratio as a function of angular distance from the cardinal meridians. Error bars indicate standard error across subjects.

\section{$\mathrm{HM} / \mathrm{VM}$ asymmetry persists at inner cortical depths}

The BOLD signal is strongly influenced by properties of the brain's vasculature. Uneven venous contributions across the brain can cause variation in BOLD magnitude (Menon et al., 1993; Kim et al., 1994; Hoogenraad et al., 1999; Kay et al., 2019). One possibility is that the meridional asymmetries we observe arise from non-uniformities in the vascular network. To investigate this possibility, we took advantage of the sub-millimeter resolution of the TDM dataset and examined HM/VM asymmetry as a function of cortical depth. Because macroscopic venous effects are larger in the superficial cortex due to large pial veins (Duvernoy et al., 1981; Turner, 2002; Polimeni et al., 2010; Kay et al., 2019), by sampling BOLD responses from deeper cortical depths, we minimize contributions from pial veins. We find that the HM/VM asymmetry is larger at the superficial depths, suggesting that part of the asymmetry may be due to differential properties in macroscopic vasculature (Figure 5). This depth effect is systematic: every subject shows higher asymmetry at the superficial depth than the middle depth. Nonetheless, there remains a substantial horizontal/vertical asymmetry at all cortical depths (Figure 5), suggesting that macroscopic vessels near the pial surface are not the entire explanation. At the innermost cortical depth sampled, which is 
least influenced by pial vessels, the HM/VM asymmetry is $26 \%$ (average across subjects) and is positive in each of the 5 subjects. The middle depths appear to have the least asymmetry. This could be due to a difference in neural responses at intermediate depths, which generally correspond to input-related cortical layers.

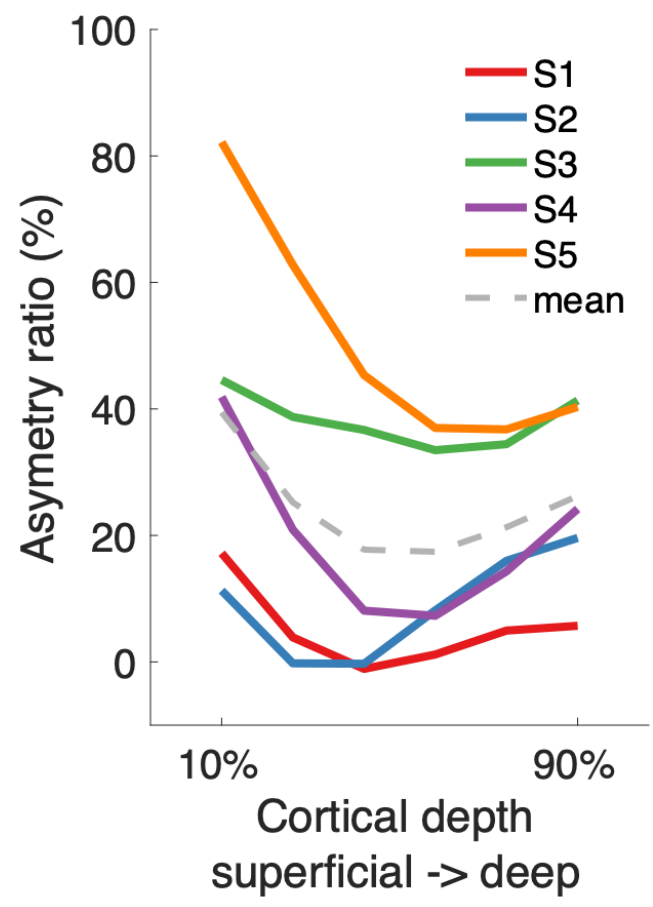

Figure 5 - BOLD asymmetry in V1 persists at inner cortical depths. We exploit the high-resolution TDM dataset to discriminate V1 BOLD responses across cortical depth and estimate response asymmetries as a function of depth (asymmetry is calculated in the same way as in Figure 3 ). The presence of asymmetry at the innermost cortical depth suggests that response asymmetries exist even with minimal contribution of large pial veins.

\section{Assessing and modeling non-neural contributions to BOLD signal magnitude}

In addition to vascular effects, other factors unrelated to neural activity evoked by the experimental manipulation may influence variation in \%BOLD across the cortical surface. These additional factors are often neglected in $\mathrm{fMRI}$ analysis pipelines. Although some of the factors are known to vary across the cortex, their influence on the BOLD signal is poorly understood. Here, we attempt to understand how these factors may induce BOLD magnitude variations. To the best of our knowledge, we are unaware of any previous study that has examined this issue in detail, especially at the level of individual voxels (or vertices) within individual subjects.

We first identified a list of possible confounding factors based on consideration of basic anatomical properties of the brain and the nature of $\mathrm{fMRI}$ measurement. These factors are cortical curvature, cortical thickness, radiofrequency (RF) coil bias, mean bias-corrected EPI signal intensity, and angle between the cortical surface and the direction of the static magnetic field B0. Each of these factors can be interpreted as spatial maps, with a value at each vertex on the cortical surface mesh. The five maps can be obtained from standard 
bioRxiv preprint doi: https://doi org/10.1101/2021.12 26.474185. this version posted December 26, 2021. The copyright holder for this preprint (which was not certified by peer review) is the author/funder, who has granted bioRxiv a license to display the preprint in perpetuity. It is made available under aCC-BY-ND 4.0 International license.

anatomical scans (T1-weighted) or from the fMRI measurements themselves without additional MRI experiments (see Methods for details). Example surface visualizations of these maps together with \%BOLD are shown in Figure 6. We hypothesize that inhomogeneities in some of these maps might explain some of the observed inhomogeneity in \%BOLD across V1.

Cortical curvature
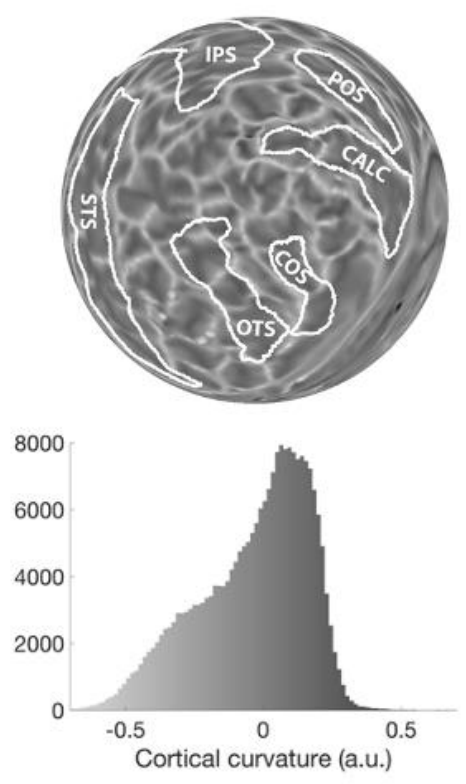

Cortical thickness
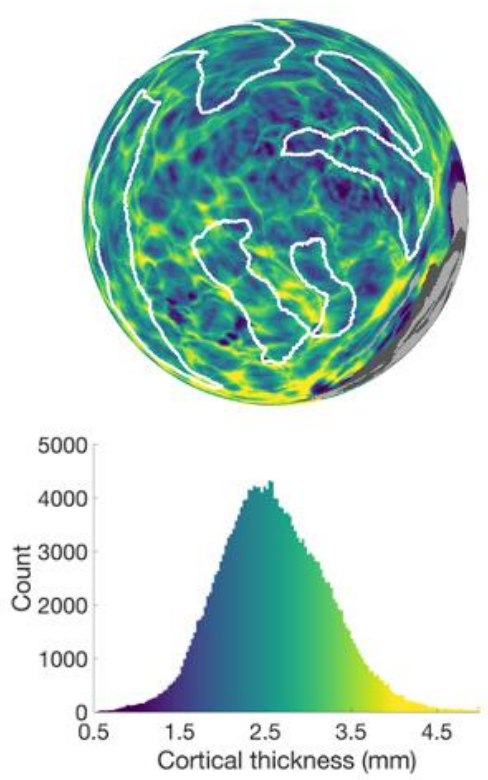

Bias-corrected EPI
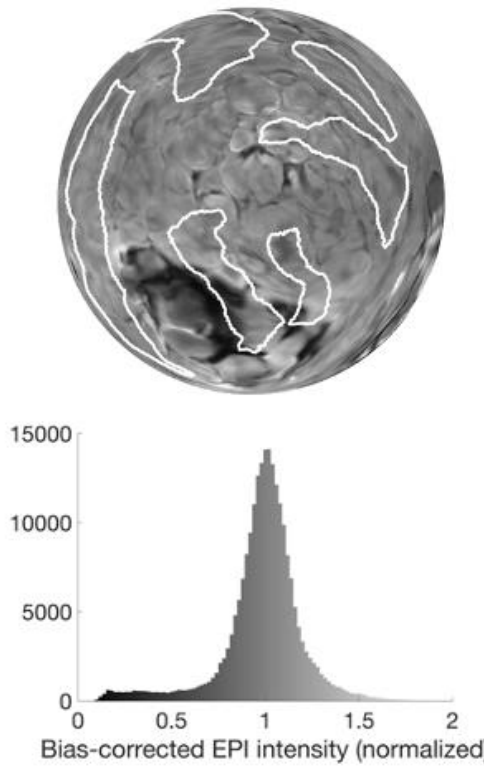

Angle with respect to $\mathrm{B} 0$
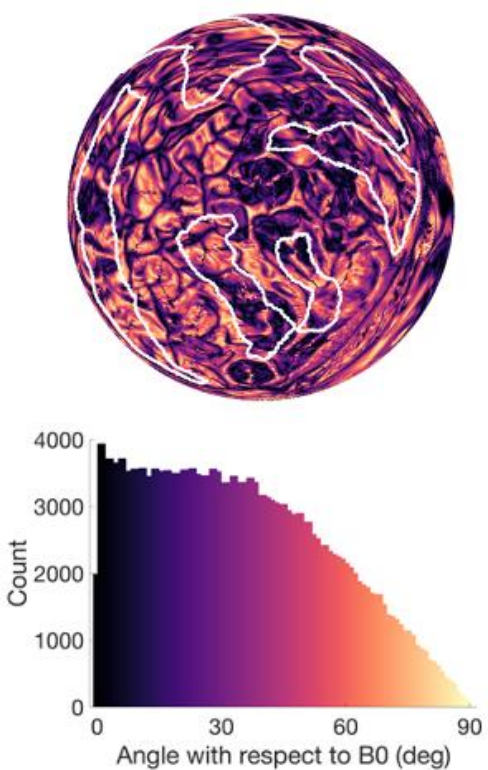

RF coil bias
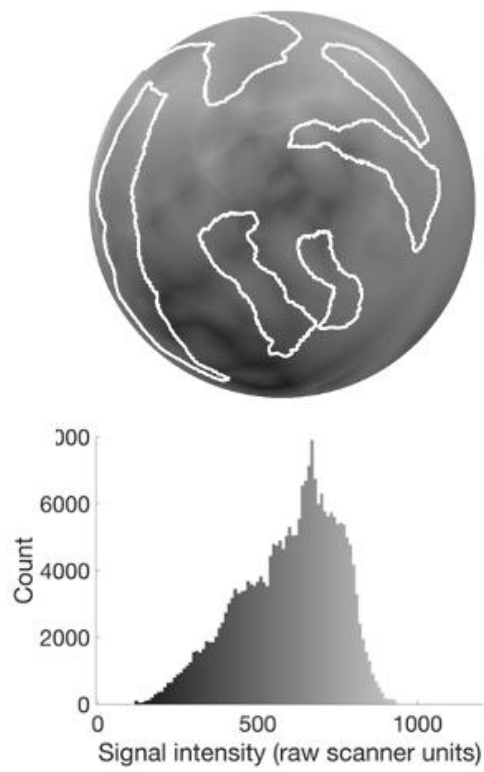

\%BOLD
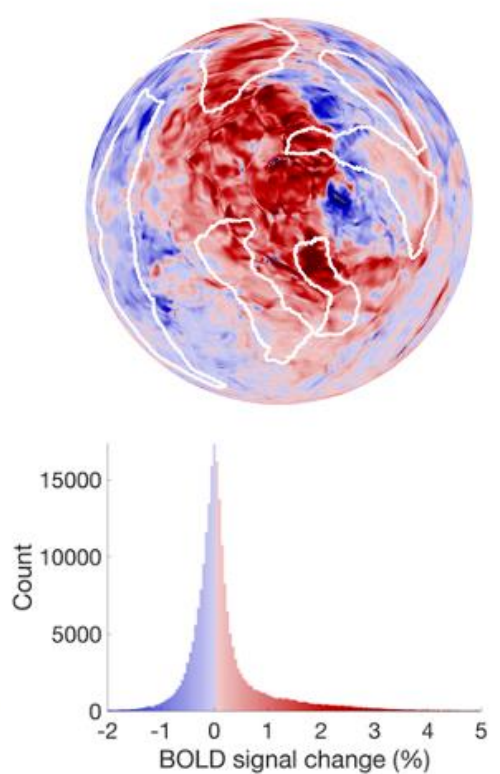

397

398

399

400

401

402

403

Figure 6 - Anatomical and acquisition factors across cortex. Each sphere shows data mapped on the left hemisphere for subject S1 in the NSD dataset. Below each surface map is a histogram of the plotted values. White outlines indicate major cortical sulci. \%BOLD represents the average response to the natural scene stimuli used in the NSD dataset. Some of the spatial variability in \%BOLD might be due to variability in the depicted non-neural factors. 
To understand the potential relationships amongst these five identified factors and \%BOLD, we first performed voxel-wise correlation analyses. For these analyses, we used the TDM dataset, as its high spatial resolution facilitates the identification of vascular effects (Kay et al., 2019). We examined data from V1-V3 where neural activity magnitudes can be expected to be relatively homogeneous given the simple contrast patterns used. In Figure 7A, we show pairwise correlations across these five quantities, as well as retinotopic polar angle preference (rescaled between 0 and 90; see Methods) and cortical depth. We find that \%BOLD correlates substantially with four factors: curvature $(r=0.26)$, thickness $(r=-0.17)$, mean bias-corrected EPI intensity $(r=-0.25)$, and depth $(r=-0.27)$. We do not find a strong correlation between \%BOLD and polar angle. Although results from Figure 3C-E, Figure $4 \mathrm{C}$ and Figure 5 suggest a strong negative correlation, the previous analysis included data only from V1. Here we analyze vertices from V1-V3 where this relationship becomes weaker $(r=$ $-0.05)$. Overall, we can summarize as follows: \%BOLD extracted from V1-V3 tends to be higher at locations that correspond to sulci, in thinner parts of the cortex, in voxels with lower mean bias-corrected EPI intensities, and at more superficial cortical depths. To assess the generality of these findings, we also examined the NSD dataset where substantially more cortex (beyond V1-V3) is activated by the natural scene images. Across the nsdgeneral ROI (drawn to cover visually responsive posterior cortex), we again find a correlation between \%BOLD and curvature $(r=0.12 ; p<0.01$, one-sample $t$-test across subjects $)$ and between \%BOLD and thickness $(r=-0.25 ; p<0.01)$. Correlation with mean bias-corrected EPI is small ( $r=-0.01$, likely due to the lower resolution of the NSD data $(1.8-\mathrm{mm})$ which tends to obscure the macrovasculature.

Examination of correlations amongst factors (excluding \%BOLD) yields additional insights. The strongest correlation that we find is between curvature and thickness $(r=-0.28)$, indicating that sulci tend to be thin. Curvature is correlated with mean bias-corrected EPI ( $r$ $=0.16)$ and with polar angle $(r=-0.19)$, and thickness is correlated with polar angle $(r=$ 0.15). Our interpretation of these effects is that venous effects tend be stronger in gyri (consistent with previous findings in Kay et al., 2019), and that the correlations related to polar angle simply reflect the tendency for horizontal meridian representations to fall on sense of non-neural influences on \%BOLD requires a broad perspective that considers 
439

440

441

442

443

444

445

446

447

448

\section{9}

450

451

452

453

454

455

456

457

458

459

460

461

462

463

464

465

466

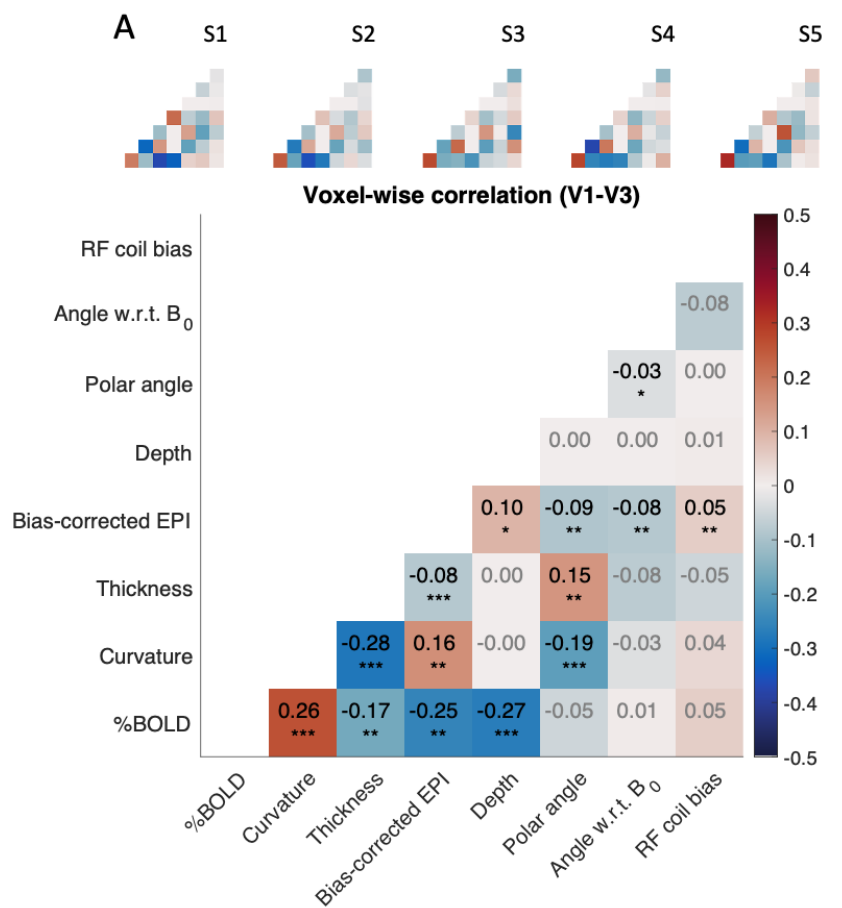

B

$$
Y=\beta_{0}+\beta_{1} X_{C}+\beta_{2} X_{T}+\beta_{3} X_{B C . E P I}
$$

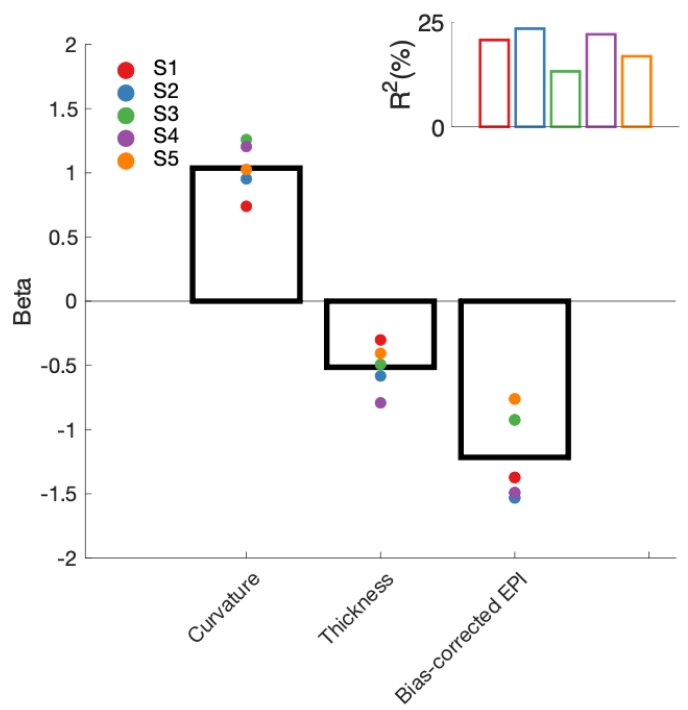

Figure 7 - Modeling variations in BOLD signal magnitude. A) Correlation (Pearson's $r$ ) between neural and non-neural factors and \%BOLD extracted from V1-V3 from the TDM dataset. Main plot shows results from data concatenated across all subjects, while inset plots show results from individual-subject data. P-values indicate significance of one sample $t$-test across subjects; ${ }^{*} p<0.05 ;{ }^{* *} p<0.01 ;{ }^{* * *} p<0.001$. B) Regression model for \%BOLD. Based on the results of panel A, we selected curvature, thickness, and mean bias-corrected EPI as the main non-neural factors that confound \%BOLD. These three factors were then used in a multiple linear regression model to predict \%BOLD (top). The amount of variance explained by the model is shown in the inset.

\section{Correcting BOLD signal magnitude for non-neural factors}

We now explore whether we can develop a statistical model to compensate for the influence of non-neural factors on \%BOLD. We operate under the assumption that any observed correlation between the factors and \%BOLD is incidental and does not reflect genuine neural activity variation. For our model, we do not use cortical depth as a factor because it is not practically accessible in standard-resolution fMRI measurements and, more importantly, because aggressively removing its contribution would clearly remove true (linear) variations in depth-dependent activity. Our model is a multiple regression model (Figure 7B, top) that uses the main factors of curvature, thickness, and mean bias-corrected EPI intensity as continuous variables and attempts to determine a weighted sum of these factors that optimally accounts for variations in \%BOLD across cortical locations (see Methods for details).

Fitting the model, we find a strong positive contribution of curvature and negative contributions of cortical thickness and EPI intensity (Figure 7B, bottom), consistent with the earlier voxel-wise correlation analyses. Estimated beta weights are fairly consistent across subjects, and the model on average across subjects explains $19 \%$ of the variance in \%BOLD. 
A multiple regression model using all 5 factors resulted in only minimally larger explained variance, $20 \%$ vs. $19 \%$, consistent with the earlier correlation analyses indicating that RF coil bias and $\mathrm{BO}$ angle bear little or no relationship with \%BOLD.

To better understand the relationship between the identified non-neural factors and \%BOLD, we construct a 2D histogram relating the model fit (BOLD prediction based on nonneural factors obtained by multiplying the design matrix and estimated beta weights) and \%BOLD (Figure 8A). This reveals a clear nonlinear relationship. To accommodate this nonlinearity, we fit a nonlinear function relating the linear model fit and \%BOLD (blue line in Figure $\mathbf{8 A}$ ). Finally, we remove the contribution of non-neural factors by dividing \%BOLD observed at each cortical location by the fit of the nonlinear model. The interpretation is that the influence of non-neural factors on \%BOLD might impose a type of 'gain' field on $\mathrm{fMRI}$ responses observed in a given experiment. Note that our method of rescaling BOLD magnitudes does not change the pattern of responses across different experimental conditions within a voxel. For example, if the response to condition $A$ is $25 \%$ higher than the response to condition $B$, this will continue to be the case after rescaling.
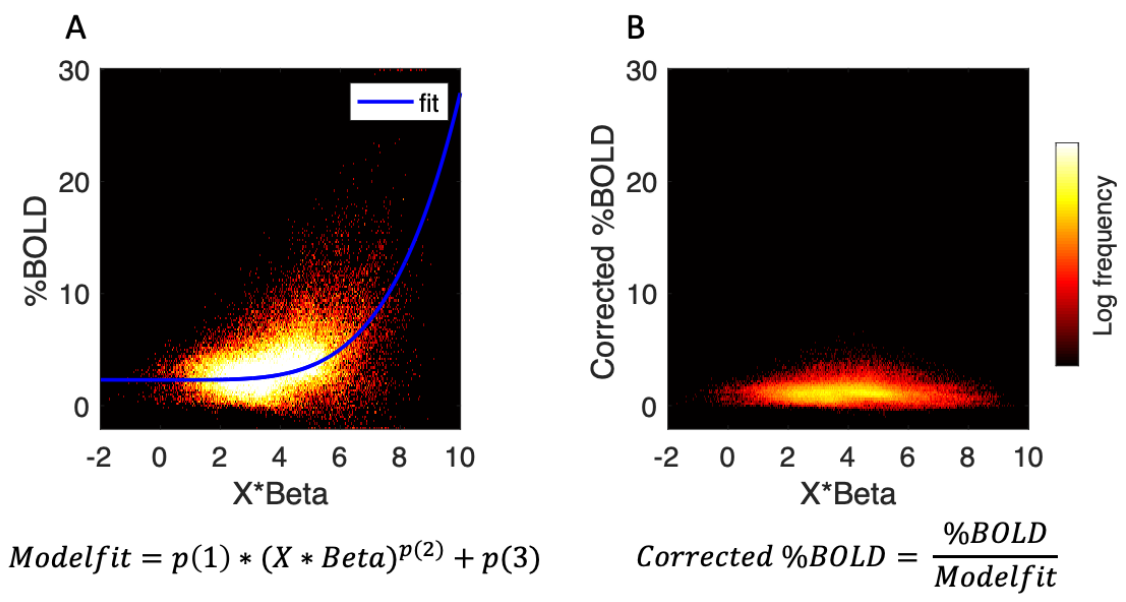

C
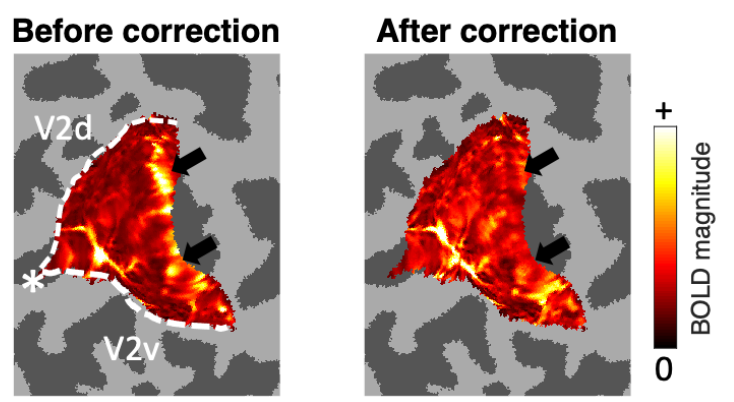

484

485

486

487

488

489

490

491
Figure 8 - Correction of V1 BOLD inhomogeneity. A) Removal of non-neural factors. First, linear combinations of non-neural factors are used to predict \%BOLD within V1-V3 using the TDM dataset. The model is fit on data concatenated from all 5 TDM subjects. The model is augmented with a nonlinear power-law function (blue line), which is controlled by a gain parameter $(p(1))$, an exponent parameter $(p(2))$, and a constant term $(p(3))$. B) Each voxel's BOLD responses are divided by the model fit, yielding the corrected \%BOLD. C) BOLD signal magnitude within V1 before and after the correction (TDM dataset, subject S3, most superficial cortical depth). Asterisk indicates the fovea and dashed lines indicate the boundary between V1 and V2. After correction, 
494 The result of the proposed correction procedure is shown in Figure 8B. We see that after the correction procedure, the distribution of BOLD response becomes flatter, indicating the efficacy of the procedure. Increased homogeneity of BOLD magnitude is also visible on the cortical surface (Figure $\mathbf{8 C}$ ). As a further check of the validity of our procedure, we quantified the homogeneity of BOLD magnitudes across V1 using the coefficient of determination (CoV; ratio of standard deviation to mean). We find that the CoV decreases from $88 \%$ before correction to $64 \%$ after correction (Figure $9 \mathrm{C}$ ). We also summarize the effect of the correction by showing the correlations between \%BOLD and non-neural factors before and after the correction (Figure 9D). Correlations after the correction are substantially reduced, though the correlation between \%BOLD and depth remains strong.

504

We now return to the experimental effect that motivated this study, namely, BOLD response asymmetries across the horizontal and vertical meridians in V1. We quantify the asymmetry before (Figure 9A) and after (Figure 9B) the correction. We find that before accounting for the non-neural factors, the asymmetry is $41.7 \%$, but after the correction, the asymmetry is reduced to $23.1 \%$. We thus suggest that some of the observed differences in BOLD response magnitudes are due to non-neural factors, but the remaining differences may reflect genuine differences in local neural activity across these subregions of V1.

512

A

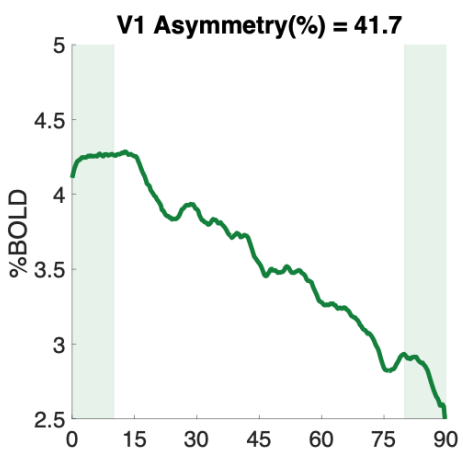

B

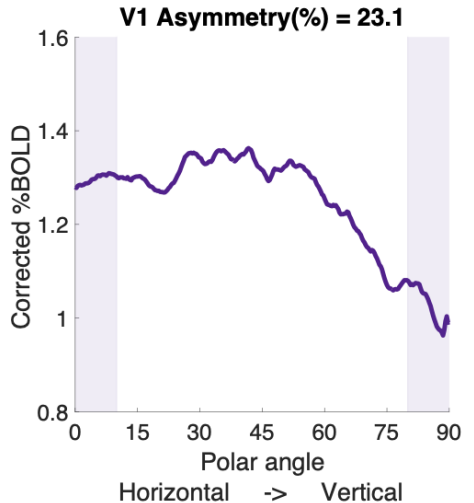

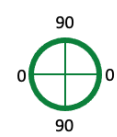

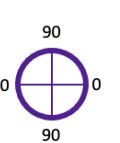

C

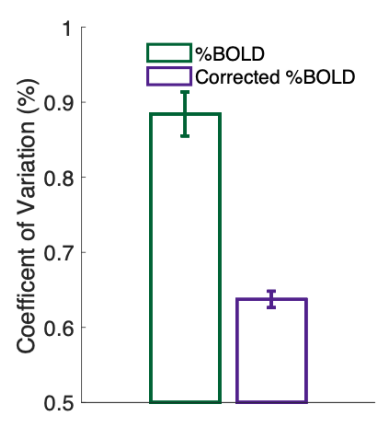

D
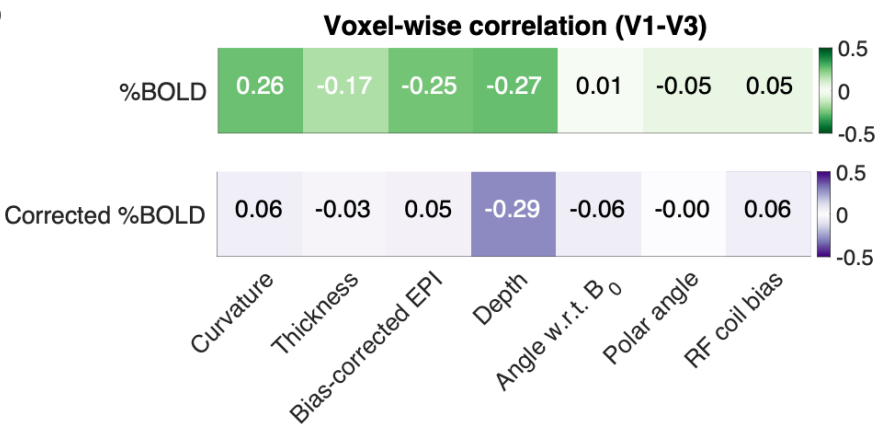

Figure 9 - The effect of BOLD inhomogeneity correction. A) Dependence of \%BOLD on polar angle in V1 before correction (TDM dataset, results summarized across subjects and depths using local linear regression). Shaded area indicates the width of the wedge used to calculate the asymmetry ratio reported in the plot title. 
B) Same as panel A, except showing results after correction. C) Coefficient of variation of \%BOLD across V1 before and after correction (error bars indicate standard error across subjects). D) Voxel-wise correlation between the various factors and \%BOLD before and after correction.

\section{Cerebral sinuses do not explain horizontal/vertical asymmetry}

522

523

524

525

526

527

528

529

530

531

532

533

534

535

536

537

538

539

540

541

542

543

544

545

546

547

548

549

550

551

552

553
The cerebral sinuses are the largest veins that drain blood away from the brain and are composed of three main branches (superior sagittal sinus, straight sinus, and transverse sinus) (Figure 10A). Due to their very large size, the cerebral sinuses introduce substantial magnetic field inhomogeneities that may result in BOLD signal dropout and/or distortion (Winawer et al., 2010; Jamison et al., 2017). One possibility is that sinus-related artifacts may be related to the observation of horizontal/vertical asymmetry. Specifically, it might be that the cerebral sinuses tend to be located near the vertical meridians, cause loss of BOLD signal, and therefore produce horizontal/vertical asymmetry. Modelling the effects of the sinus is difficult. First, both veins and sinuses result in low EPI intensity values, making them difficult to distinguish using conventional EPI data. Second, they have different effects on the BOLD signal. BOLD responses tend to be higher near small and moderately sized veins but tend to be lower (or non-existent) near the sinuses. Third, mapping the exact cortical location of the sinuses is challenging.

To assess the impact of the sinuses on our measurements, we constructed an MNI atlas of likely sinus locations (see Methods). We then mapped this atlas to the native space of individual subjects and identified surface vertices that are likely to be near the sinuses. Consistent with previous studies (Winawer et al., 2010), we find that several branches of the sinuses tend to overlap with primary visual cortex (Figure 10B) and more specifically with the representation of the vertical meridian (Figure 10C-D; results for all subjects can be found in Supplementary Figure 1). To test whether presumed signal loss from the sinuses might be responsible for horizontal-vertical asymmetry, we removed vertices close to the sinuses and re-computed horizontal-vertical asymmetry. Across different inclusion levels (removing voxels close to or further away from the sinuses), we find very little effect on the asymmetry (Figure 10E-F). Our interpretation is that the sinuses tend to co-localize with the $\mathrm{VM}$, but this correspondence is not perfect (and is variable across subjects), and there are sufficient remaining VM vertices for which to compute horizontal-vertical asymmetry. We therefore conclude that horizontal-vertical asymmetry is not a simple consequence of BOLD signal loss induced by the sinuses. 
554

555

556

557

558

559

560

561

562

563

564

565

566

567

568
A

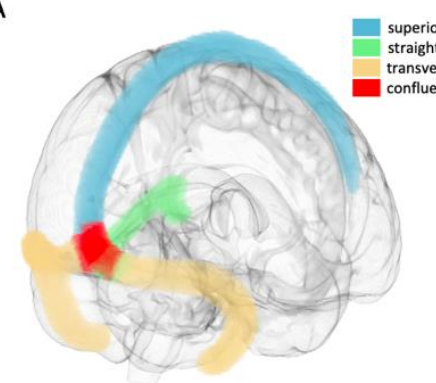

${ }_{1600}$

Polar angle in V1

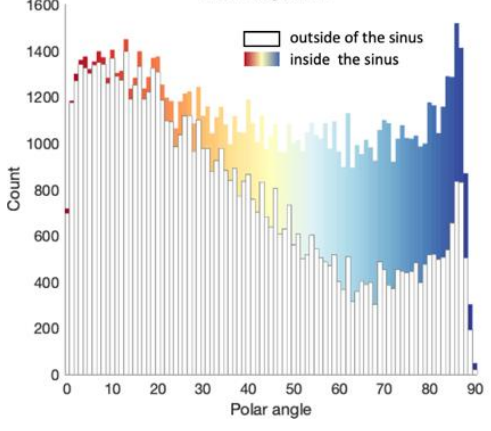

B

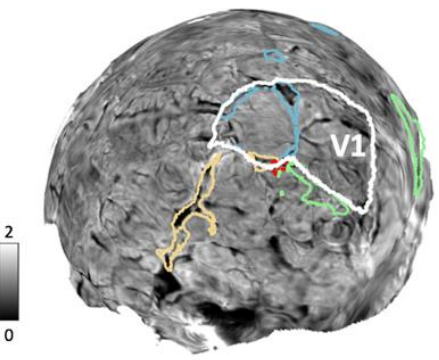

E

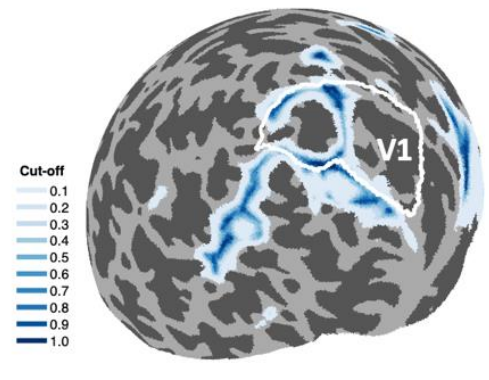

C

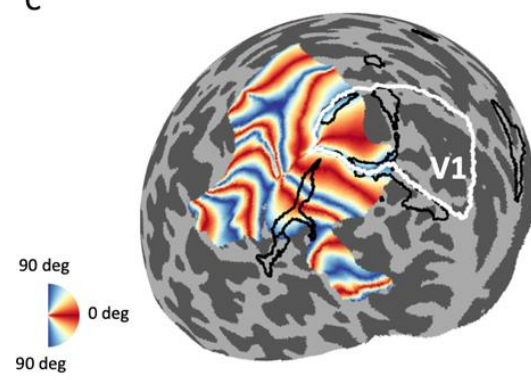

F

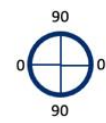

Figure 10 - Assessing the effect of the cerebral sinuses on V1 response asymmetry. A) Sinus atlas in MNI space visualized against a 3D glass brain. There are three main branches (marked by different colors) with a confluence located close to the occipital pole. B) Sinus atlas mapped to the cortical surface of subject S5 from the TDM dataset (cut-off $=0.8$ ). The underlying data are mean bias-corrected EPI intensity values. C) Same as panel B except the underlying data are polar angle estimates from the Benson atlas (thresholded at a stimulus eccentricity of $5.5 \mathrm{deg}$ ). D) Stacked histogram showing polar angle values from all subjects inside the V1 ROI (cut-off $=0.8$ ). White bins indicate locations outside of the sinus atlas; colored bins indicate locations within the sinus atlas. E) Spatial extent of sinus influence. After blurring a binary version of the sinus atlas with a 5$\mathrm{mm}$ FWHM Gaussian, we use different cut-off values to select different spatial extents of the influence of the sinuses. F) Corrected \%BOLD obtained from the TDM dataset as a function of polar angle. Here we use different cut-off values to exclude sinus-related vertices and plot the resulting corrected \%BOLD (local regression estimate; same as Figure $9 \mathrm{~A}-\mathrm{B})$. For each cut-off value we calculate the asymmetry ratio and report the value in the text inset. Shaded area indicates the width of the wedge used to calculate the asymmetry.

\section{Discussion}

In this paper, we used three publicly available datasets to assess the degree of homogeneity of BOLD signal magnitude in primary visual cortex. We found that stimulus-evoked BOLD responses, expressed as percent signal change, are up to $50 \%$ stronger along the horizontal meridian than the vertical meridian. To investigate whether these magnitude differences can be attributed to differences in local neural activity, we systematically evaluated the potential contribution of several non-neural factors to the observed effect. We found that BOLD signal magnitude correlates with cortical curvature, cortical thickness, and macrovasculature (as indexed by bias-corrected EPI intensities). Using a regression-based correction procedure, we were able to increase the homogeneity of BOLD signal magnitude and found that the meridional differences were reduced by half. 


\section{Spatial variations in BOLD magnitude}

582

583

584

585

586

587

588

589

590

591

592

593

594

595

596

597

598

599

600

601

602

603

604

605

606

607

608

609

610

611

612

613

614

615

616

617

618

619

620

621

622

623

624

This study tackles the issue of the neural basis of variation in BOLD signal magnitude. Specifically, we address variation in BOLD across cortical locations for a fixed experimental manipulation, as opposed to variation in BOLD across experimental manipulations for a fixed cortical location. The latter has been heavily studied (Heeger et al., 2000; Logothetis et al., 2001; Heeger and Ress, 2002; Logothetis and Wandell, 2004; Mishra et al., 2021), whereas the former has not yet been systematically studied to the best of our knowledge. If there are indeed non-neural factors that influence BOLD signal variation, taking this into account is critical when interpreting differences in $\mathrm{fMRI}$ responses across brain regions. We acknowledge that a challenge in understanding the neural basis of the BOLD signal is that directly comparable ground-truth measurements of neural activity are typically not available. In our analyses, we relied on the working assumption that the experimental paradigms of the three datasets (combined with suitable averaging and analysis procedures) are expected to generate relatively homogeneous patterns of neural activity in early visual cortex. Of course, this may not be exactly the case.

We note that some analyses of $\mathrm{fMRI}$ data are insensitive to spatial variation of BOLD magnitude. For example, population receptive field models incorporate an explicit gain parameter that is distinct from other parameters (such as receptive field position) in the model. As such, such approaches can discount the gain observed at a given voxel. However, in general, conventional approaches to $\mathrm{FMRI}$ analysis in which the size of the BOLD response is used for inference (including subtraction-based analyses) are susceptible to the influence of non-neural factors on BOLD signal magnitude.

\section{Non-neural factors that affect BOLD magnitude}

Cortical anatomy. We find that cortical curvature and cortical thickness correlate with BOLD signal magnitude (see Figure 7A). It is known that many anatomical properties vary with thickness and with curvature (Jiang et al., 2021): (i) total neuron count is higher in gyri than it is in sulci (Hilgetag and Barbas, 2005), (ii) gyri tend to be thicker than sulci (Welker, 1990; Hilgetag and Barbas, 2005), (iii) venous effects (resulting in higher BOLD signal amplitude) are more prominent in gyri than they are in sulci (Kay et al., 2019); and (iv) there may even be intrinsic causal relationships between curvature and thickness during anatomical development (Hilgetag and Barbas, 2005).

However, the exact anatomical and biophysical mechanisms that might link curvature and thickness to BOLD signal magnitudes and whether they reflect purely non-neural sources of variance or might be linked to variations in neural activity is largely unknown, to our knowledge. This is an important issue for future research. Here, we operate under the working assumption that correlations between the BOLD signal and cortical curvature or thickness reflect incidental factors unrelated to local neural activity. We therefore assume that a correction which removes their influence from the BOLD signal is desirable. 
Cerebral sinuses. One possible explanation of the horizontal/vertical asymmetry pertains to the severe magnetic field inhomogeneities introduced by the cerebral sinuses. Consistent with Winawer et al. (2010; their figure 10), we find that the transverse sinus is close to the upper vertical meridian of V1 (ventral bank of calcarine sulcus) and that vertices near the sinus tend to be darker (lower mean EPI). Although the sinus is known to corrupt the fMRI signal (Winawer et al, 2010), we found that removing voxels near the sinus did not eliminate the horizontal/vertical asymmetry. The atlas we create in this paper helps identify cortical regions likely to be influenced by the cerebral sinuses. Note that the location of the sinuses is fairly consistent across subjects in volume-based MNI space but is quite variable across subjects when projecting to individual subjects' native surfaces (see Supplementary Figure 1). We expect this atlas will prove valuable for studying sinus-related regions in future studies.

Orientation of pial veins. It has been reported that regions where the cortical surface is oriented perpendicular to the main magnetic field produce lower BOLD signal than regions where the surface is oriented parallel (Gagnon et al., 2015; Fracasso et al., 2018). The proposed explanation is that this effect is caused by the orientation of pial veins, which lie parallel to the cortical surface. Our analyses did not replicate this result and indicated little relationship between BOLD magnitude and BO angle (see Figure 7A). One possible explanation could be related to the pre-processing pipeline for the TDM dataset, in which fMRI signals were sampled in the gray matter at $10 \%$ or more of the cortical thickness away from the pial surface. This may have dampened effects related to pial veins, though a detailed examination would be necessary to resolve these discrepancies.

RF coil effects. Due to cortical folding, gyri tend to be closer to the RF coil than sulci. Locations that are further from the coil might have lower mean signal intensities and therefore lower SNR (Srirangarajan et al., 2021), but this should not affect BOLD magnitudes expressed in terms of percent signal change. We are not aware of any mechanism that would alter the percent signal change in brain locations that are further away from the RF coil (e.g., lower BOLD responses in sulci compared to gyri). Indeed, we did not find any relationship between RF coil bias and BOLD magnitude (see Figure 7A).

\section{Correction for the impact of non-neural factors}

Our results show that voxel-wise \%BOLD is likely contaminated by several non-neural factors. To account for these factors, we developed a regression-based correction method. The goal of this method was to introduce a simple, data-driven approach that can be applied irrespectively of the specific experiment or brain region that is under consideration. The underlying premise of the method is that by removing the contribution of non-neural factors, the resulting measures would constitute a better representation of the underlying neural activity. After application of the method, we found that \%BOLD becomes more homogenous and correlations between \%BOLD and non-neural factors become significantly reduced. Thus, our results indicate that some variation in \%BOLD that might be interpreted as change in neural activity likely reflects the variation of non-neural factors. We believe the results presented in this paper constitute a first step towards developing a cogent strategy for compensating for non-neural biases in BOLD signal magnitudes. 
672 There are other methods that can be used to suppress the contribution of non-neural

673 factors to BOLD signal magnitudes. By identifying early and late components of evoked 674 hemodynamic responses, a temporal decomposition method can be used to estimate BOLD 675 response components more closely linked to the microvasculature, which presumably more 676 closely reflect local neural activity (Kay et al., 2020). Another analysis method focuses on BOLD fluctuations where estimates of slow oscillations $(<0.1 \mathrm{~Hz})$ are used to suppress vascular-related effects (Kazan et al., 2016). Similarly, some methods use the amplitude of fluctuations in resting-state data to rescale the BOLD signal (Di et al., 2013; Guidi et al., 2020). Finally, acquisition methods, such as spin-echo pulse sequences, can be used to suppress unwanted venous effects. Note that all these methods concern effects of the macrovasculature, but systematic biases in BOLD signal magnitudes may in theory persist even if BOLD responses were restricted to the microvasculature. Further investigation is necessary to resolve these possibilities.

685

686

More generally, our findings here are part of a broader effort to make meaningful 687 inferences about the underlying biology from MRI measurements. While the pixel intensity of most MR images is expressed in arbitrary units, there has been some progress in removing the influence of unwanted factors to quantify specific properties of the measured tissue. For example, using anatomical imaging, one can estimate the macromolecular tissue volume within a voxel (Mezer et al., 2013), a quantity that has clinical relevance for neurodegenerative diseases. Our study is a step towards linking BOLD amplitude more directly to neural activity.

\section{Author Contributions}

695

696

697

698

699

700

701

702

703

704

705

706

707

708

709

710

711

712

713
J.W.K, K.K. and J.W. conceived and designed this study, K.K. acquired the data. J.W.K and K.J. performed data analyses, with K.K. and J.W. providing guidance. J.W.K., J.W, O.F.G and K.K. wrote and edited the manuscript. 
bioRxiv preprint doi: https://doi org/10.1101/2021.12.26.474185. this version posted December 26, 2021. The copyright holder for this preprint (which was not certified by peer review) is the author/funder, who has granted bioRxiv a license to display the preprint in perpetuity. It is made available under aCC-BY-ND 4.0 International license.

714

\begin{tabular}{|l|l|l|l|}
\hline Dataset & TDM & NSD & HCP \\
\hline Field strength & $7 T$ & $7 T$ & $7 T$ \\
\hline TR & $2200 \mathrm{~ms}$ & $1600 \mathrm{~ms}$ & $1000 \mathrm{~ms}$ \\
\hline TE & $22.4 \mathrm{~ms}$ & $22.0 \mathrm{~ms}$ & $22.2 \mathrm{~ms}$ \\
\hline Flip angle & 80 & 62 & 45 \\
\hline Number of slices & 84 & 84 & 85 \\
\hline Matrix size & $200 \times 162$ & $120 \times 120$ & $130 \times 130$ \\
\hline Field of view spatial & $\begin{array}{l}160 \mathrm{~mm} \times 129.6 \\
\mathrm{~mm}\end{array}$ & $216 \mathrm{~mm} \times 216 \mathrm{~mm}$ & $208 \mathrm{~mm} \times 208 \mathrm{~mm}$ \\
\hline $\begin{array}{l}\text { Nominal } \\
\text { resolution }\end{array}$ & 2 & $1.8 \mathrm{~mm}$ & $1.6 \mathrm{~mm}$ \\
\hline Multiband factor & 3 & 3 & 5 \\
\hline iPAT factor & $6 / 8$ & 2 & 2 \\
\hline Partial Fourier & $7 / 8$ & $7 / 8$ \\
\hline
\end{tabular}
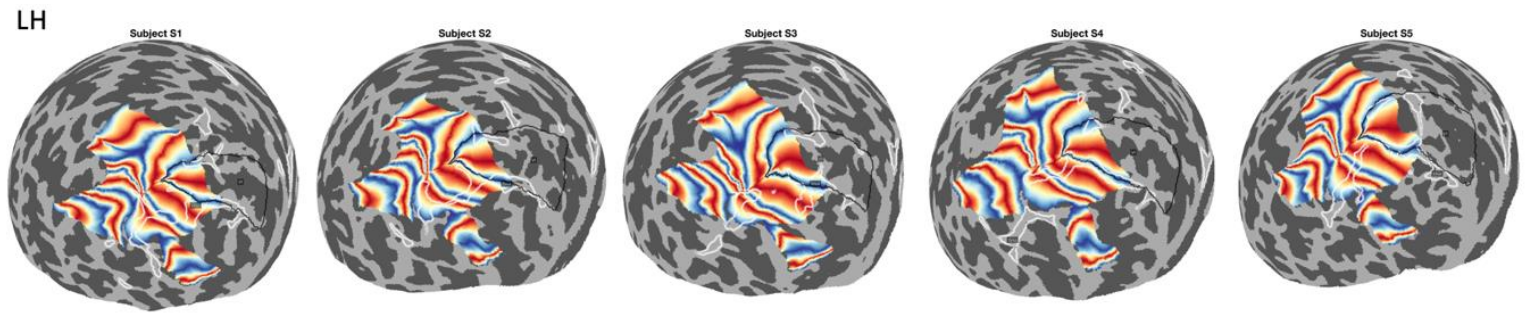

$\mathrm{RH}$
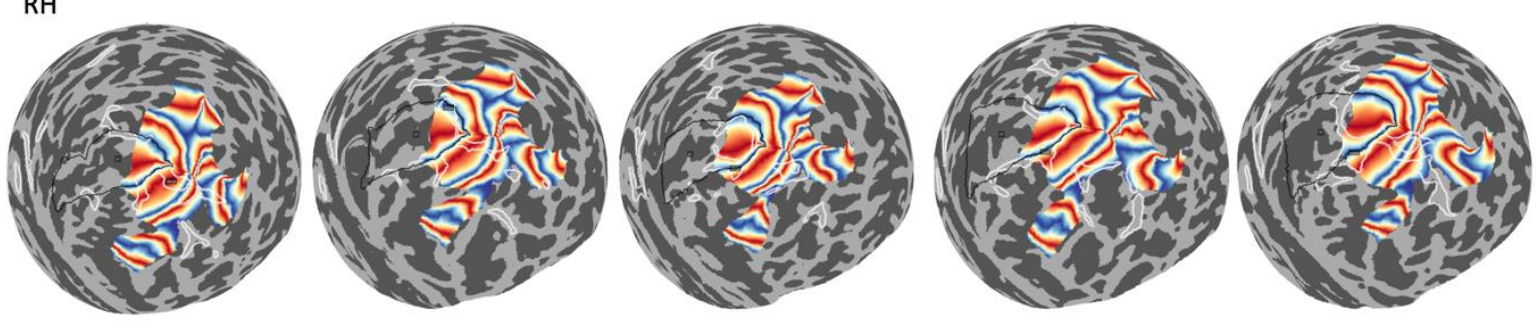

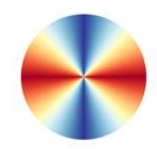

Supplementary Figure 1 - The location of the cerebral sinuses relative to primary visual cortex. Polar angle map (see legend) derived from Benson's atlas (Benson et al., 2014) is shown on a spherical cortical surface for each subject and hemisphere in the TDM dataset. Light gray and dark gray indicate gyri and sulci, respectively. The location of V1 (obtained from the atlas) is outlined in black. The estimated location of the sinuses is outlined in white. In some subjects, the sinuses are positioned near the dorsal and ventral boundaries of V1 (corresponding to the vertical meridian), but in other subjects, the sinuses do not align well with these boundaries. 
Allen EJ, St-Yves G, Wu Y, Breedlove JL, Prince JS, Dowdle LT, Nau M, Caron B, Pestilli F, Charest I, Hutchinson JB, Naselaris T, Kay K (2021) A massive 7T fMRI dataset to bridge cognitive neuroscience and artificial intelligence. Nature Neuroscience.

Arthurs OJ, Williams EJ, Carpenter TA, Pickard JD, Boniface SJ (2000) Linear coupling between functional magnetic resonance imaging and evoked potential amplitude in human somatosensory cortex. Neuroscience 101:803-806.

Attwell D, ladecola C (2002) The neural basis of functional brain imaging signals. Trends Neurosci 25:621-625.

Benson NC, Winawer J (2018) Bayesian analysis of retinotopic maps. Elife 7.

Benson NC, Butt OH, Brainard DH, Aguirre GK (2014) Correction of distortion in flattened representations of the cortical surface allows prediction of $\mathrm{V} 1-\mathrm{V} 3$ functional organization from anatomy. PLoS Comput Biol 10:e1003538.

Benson NC, Jamison KW, Arcaro MJ, Vu AT, Glasser MF, Coalson TS, Van Essen DC, Yacoub E, Ugurbil K, Winawer J, Kay K (2018) The Human Connectome Project 7 Tesla retinotopy dataset: Description and population receptive field analysis. $\mathrm{J}$ Vis 18:23.

Britten KH, Shadlen MN, Newsome WT, Movshon JA (1993) Responses of neurons in macaque MT to stochastic motion signals. Vis Neurosci 10:1157-1169.

Dale AM, Fischl B, Sereno MI (1999) Cortical Surface-Based Analysis: I. Segmentation and Surface Reconstruction. Neurolmage 9:179-194.

Di X, Kannurpatti SS, Rypma B, Biswal BB (2013) Calibrating BOLD fMRI activations with neurovascular and anatomical constraints. Cereb Cortex 23:255-263.

Dumoulin SO (2017) Layers of Neuroscience. Neuron 96:1205-1206.

Dumoulin SO, Wandell BA (2008) Population receptive field estimates in human visual cortex. Neuroimage 39:647-660.

Dumoulin SO, Fracasso A, van der Zwaag W, Siero JCW, Petridou N (2018) Ultra-high field MRI: Advancing systems neuroscience towards mesoscopic human brain function. Neuroimage 168:345-357.

Duvernoy HM, Delon S, Vannson JL (1981) Cortical blood vessels of the human brain. Brain Res Bull 7:519-579.

Fischl B, Dale AM (2000) Measuring the thickness of the human cerebral cortex from magnetic resonance images. Proc Natl Acad Sci U S A 97:11050-11055.

Fracasso A, Petridou N, Dumoulin SO (2016a) Systematic variation of population receptive field properties across cortical depth in human visual cortex. Neuroimage 139:427438.

Fracasso A, Luijten PR, Dumoulin SO, Petridou N (2018) Laminar imaging of positive and negative BOLD in human visual cortex at 7T. Neuroimage 164:100-111.

Fracasso A, van Veluw SJ, Visser F, Luijten PR, Spliet W, Zwanenburg JJM, Dumoulin SO, Petridou N (2016b) Lines of Baillarger in vivo and ex vivo: Myelin contrast across lamina at 7T MRI and histology. Neuroimage 133:163-175.

Gagnon L, Sakadzic S, Lesage F, Pouliot P, Dale AM, Devor A, Buxton RB, Boas DA (2016) Validation and optimization of hypercapnic-calibrated fMRI from oxygen-sensitive two-photon microscopy. Philos Trans R Soc Lond B Biol Sci 371.

Gagnon L, Sakadzic S, Lesage F, Musacchia JJ, Lefebvre J, Fang Q, Yucel MA, Evans KC, Mandeville ET, Cohen-Adad J, Polimeni JR, Yaseen MA, Lo EH, Greve DN, Buxton RB, Dale AM, Devor A, Boas DA (2015) Quantifying the microvascular origin of BOLD-fMRI from first principles with two-photon microscopy and an oxygen-sensitive nanoprobe. J Neurosci 35:3663-3675.

Gorno-Tempini ML, Hutton C, Josephs O, Deichmann R, Price C, Turner R (2002) Echo time dependence of BOLD contrast and susceptibility artifacts. Neuroimage 15:136-142.

Guidi M, Huber L, Lampe L, Merola A, Ihle K, Moller HE (2020) Cortical laminar resting-state signal fluctuations scale with the hypercapnic blood oxygenation level-dependent response. Hum Brain Mapp 41:2014-2027. 
Heeger DJ, Ress D (2002) What does fMRI tell us about neuronal activity? Nature Reviews Neuroscience 3:142-151.

Heeger DJ, Huk AC, Geisler WS, Albrecht DG (2000) Spikes versus BOLD: what does neuroimaging tell us about neuronal activity? Nat Neurosci 3:631-633.

Herman MC, Cardoso MMB, Lima B, Sirotin YB, Das A (2017) Simultaneously estimating the task-related and stimulus-evoked components of hemodynamic imaging measurements. Neurophotonics 4:031223.

Hilgetag CC, Barbas H (2005) Developmental mechanics of the primate cerebral cortex. Anat Embryol (Berl) 210:411-417.

Hoogenraad FG, Hofman MB, Pouwels PJ, Reichenbach JR, Rombouts SA, Haacke EM (1999) Sub-millimeter fMRI at 1.5 Tesla: correlation of high resolution with low resolution measurements. J Magn Reson Imaging 9:475-482.

Jamison K, Vizioli L, Zhang R, Tao J, Winawer J, Kay K (2017) A tool for automatic identification of cerebral sinuses and corresponding artifacts in fMRI. Journal of Vision 17:295-295.

Jiang X, Zhang T, Zhang S, Kendrick KM, Liu T (2021) Fundamental functional differences between gyri and sulci: implications for brain function, cognition, and behavior. Psychoradiology 1:23-41.

Kay K, Jamison KW, Zhang RY, Ugurbil K (2020) A temporal decomposition method for identifying venous effects in task-based fMRI. Nat Methods 17:1033-1039.

Kay K, Jamison KW, Vizioli L, Zhang R, Margalit E, Ugurbil K (2019) A critical assessment of data quality and venous effects in sub-millimeter fMRI. Neuroimage 189:847-869.

Kay KN, Winawer J, Mezer A, Wandell BA (2013a) Compressive spatial summation in human visual cortex. J Neurophysiol 110:481-494.

Kay KN, Rokem A, Winawer J, Dougherty RF, Wandell BA (2013b) GLMdenoise: a fast, automated technique for denoising task-based fMRI data. Front Neurosci 7:247.

Kazan SM, Mohammadi S, Callaghan MF, Flandin G, Huber L, Leech R, Kennerley A, Windischberger C, Weiskopf N (2016) Vascular autorescaling of fMRI (VasA fMRI) improves sensitivity of population studies: A pilot study. Neuroimage 124:794-805.

Kim SG, Hendrich K, Hu X, Merkle H, Ugurbil K (1994) Potential pitfalls of functional MRI using conventional gradient-recalled echo techniques. NMR Biomed 7:69-74.

Koopmans PJ, Barth M, Orzada S, Norris DG (2011) Multi-echo fMRI of the cortical laminae in humans at 7 T. Neuroimage 56:1276-1285.

Lee JH, Durand R, Gradinaru V, Zhang F, Goshen I, Kim DS, Fenno LE, Ramakrishnan C, Deisseroth K (2010) Global and local fMRI signals driven by neurons defined optogenetically by type and wiring. Nature 465:788-792.

Logothetis NK (2002) The neural basis of the blood-oxygen-level-dependent functional magnetic resonance imaging signal. Philos Trans R Soc Lond B Biol Sci 357:10031037.

Logothetis NK (2008) What we can do and what we cannot do with fMRI. Nature 453:869878.

Logothetis NK, Wandell BA (2004) Interpreting the BOLD signal. Annu Rev Physiol 66:735769.

Logothetis NK, Pauls J, Augath M, Trinath T, Oeltermann A (2001) Neurophysiological investigation of the basis of the fMRI signal. Nature 412:150-157.

Menon RS, Ogawa S, Tank DW, Ugurbil K (1993) Tesla gradient recalled echo characteristics of photic stimulation-induced signal changes in the human primary visual cortex. Magn Reson Med 30:380-386.

Mezer A, Yeatman JD, Stikov N, Kay KN, Cho N-J, Dougherty RF, Perry ML, Parvizi J, Hua LH, Butts-Pauly K, Wandell BA (2013) Quantifying the local tissue volume and composition in individual brains with magnetic resonance imaging. Nat Med 19:16671672. 
Mishra A, Hall CN, Howarth C, Freeman RD (2021) Key relationships between non-invasive functional neuroimaging and the underlying neuronal activity. Philosophical Transactions of the Royal Society B: Biological Sciences 376:20190622.

Polimeni JR, Fischl B, Greve DN, Wald LL (2010) Laminar analysis of 7T BOLD using an imposed spatial activation pattern in human V1. Neuroimage 52:1334-1346.

Rees G, Friston K, Koch C (2000) A direct quantitative relationship between the functional properties of human and macaque V5. Nature Neuroscience 3:716-723.

Self MW, van Kerkoerle T, Goebel R, Roelfsema PR (2019) Benchmarking laminar fMRI: Neuronal spiking and synaptic activity during top-down and bottom-up processing in the different layers of cortex. Neuroimage 197:806-817.

Sethian JA (1996) A fast marching level set method for monotonically advancing fronts. Proceedings of the National Academy of Sciences 93:1591.

Siero JC, Hermes D, Hoogduin H, Luijten PR, Ramsey NF, Petridou N (2014) BOLD matches neuronal activity at the mm scale: a combined 7T fMRI and ECoG study in human sensorimotor cortex. Neuroimage 101:177-184.

Sirotin YB, Das A (2009) Anticipatory haemodynamic signals in sensory cortex not predicted by local neuronal activity. Nature 457:475-479.

Srirangarajan T, Mortazavi L, Bortolini T, Moll J, Knutson B (2021) Multi-band FMRI compromises detection of mesolimbic reward responses. Neurolmage 244:118617.

Turner R (2002) How much cortex can a vein drain? Downstream dilution of activationrelated cerebral blood oxygenation changes. Neuroimage 16:1062-1067.

van der Zwaag W, Francis S, Head K, Peters A, Gowland P, Morris P, Bowtell R (2009) fMRI at 1.5, 3 and 7 T: characterising BOLD signal changes. Neuroimage 47:1425-1434.

van Dijk JA, Fracasso A, Petridou N, Dumoulin SO (2020) Linear systems analysis for laminar fMRI: Evaluating BOLD amplitude scaling for luminance contrast manipulations. Sci Rep 10:5462.

Van Essen DC et al. (2012) The Human Connectome Project: a data acquisition perspective. Neuroimage 62:2222-2231.

Welker W (1990) Why Does Cerebral Cortex Fissure and Fold? In: Cerebral Cortex: Comparative Structure and Evolution of Cerebral Cortex, Part II (Jones EG, Peters A, eds), pp 3-136. Boston, MA: Springer US.

Winawer J, Horiguchi H, Sayres RA, Amano K, Wandell BA (2010) Mapping hV4 and ventral occipital cortex: the venous eclipse. J Vis 10:1.

Yu X, Qian C, Chen DY, Dodd SJ, Koretsky AP (2014) Deciphering laminar-specific neural inputs with line-scanning fMRI. Nat Methods 11:55-58.

Zimmermann J, Goebel R, De Martino F, van de Moortele PF, Feinberg D, Adriany G, Chaimow D, Shmuel A, Ugurbil K, Yacoub E (2011) Mapping the organization of axis of motion selective features in human area MT using high-field fMRI. PLoS One 6:e28716. 